\title{
Influence of diet with different lipid composition on neutrophil chemiluminescence and disease activity in patients with rheumatoid arthritis
}

\author{
M MAGARO', ${ }^{1}$ L ALTOMONTE ${ }^{1}$, A ZOLI, ${ }^{1}$ L MIRONE ${ }^{1}$ P DE SOLE, ${ }^{2}$ \\ G DI MARIO,2 S LIPPA, AND A ORADEI ${ }^{2}$
}

From the ${ }^{1}$ Internal Medicine Department, Rheumatology Division, and the ${ }^{2}$ Clinical Chemistry Department, Catholic University, Rome, Italy

SUMMARY Neutrophil chemiluminescence was determined in patients with active rheumatoid arthritis. Twelve patients were randomly assigned either to a diet high in polyunsaturated fatty acids supplemented with eicosapentaenoic and docosahexaenoic acids or to a diet high in saturated fatty acids. A correlation with clinical and laboratory parameters is also reported. No statistical difference was observed in neutrophil chemiluminescence and in clinical parameters in the group of patients treated with a diet high in saturated fatty acids. Fish oil ingestion resulted in subjective alleviation of active rheumatoid arthritis and reduction of neutrophil chemiluminescence. This study corroborates the hypothesis of an anti-inflammatory role for polyunsaturated fatty acids in patients with chronic inflammatory diseases.

Dietary composition has been shown to influence the immune response both in animals and in humans. ${ }^{1}$ Therefore dietary manipulations may form the basis for a nutritional approach to treating autoimmune disorders.

The regulatory effect of dietary lipid composition on many biological processes is well known. ${ }^{2-4}$ The ratio polyunsaturated:saturated fatty acids (P:S ratio) and the n-3 fatty acid amount are the important factors. Whereas the P:S ratio regulates the concentration of plasma cholesterol, ${ }^{5}$ the $n-3$ fatty acids (eicosapentaenoate 20:5, docosahexaenoate 22:6) are mainly involved in the regulation of arachidonate metabolism. ${ }^{6}$

The main source of eicosapentaenoic acid and docosahexaenoic acid is fish derived oil and an adequate dietary content seems to be $2-5 \mathrm{~g} /$ day.

The relation between dietary factors and rheumatoid arthritis is becoming more evident. ${ }^{78}$ Furthermore, patients with rheumatoid arthritis (RA) show a notable increase of arachidonate metabolism via lipoxygenase, leading to leucotrienes B4 or D4. ${ }^{9}$

Recently, Lee et al presented evidence for an inhibitory effect on polymorphonuclear activity of a diet rich in $\mathrm{n}-3$ fatty acids. ${ }^{10}$

Accepted for publication 5 March 1988.

Correspondence to Professor Mario Magaro', Istituto di Clinica Medica, Universita' Cattolica del Sacro Cuore, Largo A Gemelli 8, 00168 Rome, Italy.
Neutrophils stimulated by particulate or soluble stimuli generate a photon emission process (chemiluminescence) that is associated with NADPHoxidase activation. Chemiluminescence can be easily measured either with purified cells or with whole blood. ${ }^{11} 12$

The aim of this study was to test the effect of a diet rich in eicosapentaenoic and docosahexaenoic acids on neutrophil chemiluminescence in a group of patients with active rheumatoid arthritis. In addition, a correlation between clinical and laboratory parameters is reported.

\section{Patients and methods}

Twelve female patients with definite or classical rheumatoid arthritis as defined by the American Rheumatism Association (ARA) diagnostic criteria ${ }^{13}$ were studied. All patients had active disease as defined by the following criteria: morning stiffness of at least 30 minutes' duration, six or more tender joints, three or more swollen joints, and an erythrocyte sedimentation rate of at least $30 \mathrm{~mm} / \mathrm{h}$.

No patient had received systemic steroids or immunosuppressive or disease modifying drugs in the three months before enrolment.

The patients were randomly divided into two groups and assigned to a one month isoenergetic regimen of either a diet high in saturated fatty acids 
(P:S ratio 1.33) (group $\mathrm{A}$ ) or to a diet high in polyunsaturated fatty acids (P:S ratio 5.0) with a daily consumption of nine capsules of Max Epa (group B) (Table 1).

Max Epa consists mainly of triglycerides in which $34 \%$ of the total fatty acids and $86 \%$ of the polyunsaturated fatty acids are eicosapentaenoic acid and docosahexaenoic acid; the arachidonic acid precursor, linoleic acid, constitutes less than $2 \%$ of the fatty acids and arachidonic acid is not detectable. The amount of Max Epa orally administrated was equivalent to $1.6 \mathrm{~g}$ of eicosapentaenoic acid, $1 \cdot 1$ $\mathrm{g}$ of docosahexaenoic acid, and to $339 \mathrm{~kJ}$ daily.

All the patients were maintained on a stable dosage of non-steroidal anti-inflammatory drug. Each patient was assessed before enrolling in the study and after 30 days.

Clinical evaluation included duration of morning stiffness (in minutes), grip strength, and Ritchie's index.

At each assessment fasting blood samples were collected from the antecubital veins of patients for measurement of neutrophil chemiluminescence and for routine laboratory assessment.

Chemiluminescence was measured in whole blood as reported by De Sole et al ${ }^{12}$ with some modifications, using a commercial kit (Fagolux; Bouty, Milan, Italy). Briefly, $50 \mu \mathrm{l}$ of 1:200 diluted blood was added to $1.0 \mathrm{ml}$ final volume of the luminescent medium (Krebs-Ringer phosphate medium pH 7.4 with $0.3 \mathrm{mM} \mathrm{Ca}{ }^{++}, 5.5 \mathrm{mM}$ glucose, and $20 \mu \mathrm{M}$ luminol). Chemiluminescence was monitored at seven-minute intervals after addition of opsonised zymosan in a Packard Picolite luminometer, model 6500. Specific activity (counts per minute/ polymorphonuclear cells (cpm/PMN)) was obtained by dividing the maximum chemiluminescence activity (cpm) by the number of neutrophils (PMN) present in the test vial.

Results are expressed as mean (SE). Student's $t$ test was used for statistical evaluation.

Table 1 Baseline characteristics of patients in groups $\boldsymbol{A}$ and $B$

\begin{tabular}{lcl}
\hline & Group A & Group B \\
\hline $\begin{array}{l}\text { Mean age in years (range) } \\
\text { Mean disease duration in years }\end{array}$ & $37(20-55)$ & $36(20-50)$ \\
$\quad$ (range) & $2 \cdot 6(1-5)$ & $2 \cdot 1(1-5)$ \\
ARA class (\%) & 50 & 16 \\
I & 16 & 50 \\
II & 34 & 34 \\
III & 66 & 66 \\
Radiological stage (\%) & 34 & 34 \\
II & & \\
III & & \\
\hline
\end{tabular}

\section{Results}

No statistical differences in clinical variables were observed between the two groups of patients at the beginning of the study.

No significant differences in grip strength, Rit $\frac{\bar{C}}{0}$ chie's index, or morning stiffness were observed in group $A$ patients at the end of the study aso compared with the basal values (Fig. 1).

In group $B$ patients a tendency towards benefit? was observed for each clinical variable measured. $A \vec{P}$ significant decrease as compared with the basat value was observed in Ritchie's index after 30 days $(10.6(3.48) v 17.2(3.38) ; \mathrm{p}<0.01)$ and in morning stiffness $(22(8.45) v 33(7.34) \mathrm{min} ; \mathrm{p}<0.01)$ while $a$ significant increase was recorded in grip strengtta $(136(12.88) v 116(13.26) \mathrm{mmHg} ; \mathrm{p}<0.01)$ after 30 days (Fig. 2). Furthermore, a significant differencè between the two groups of patients at the end of the study was observed in Ritchie's index (10.6 (3.48) if $21.4(3.2) ; \mathrm{p}<0.005)$, in morning stiffness $(22(8.45)$

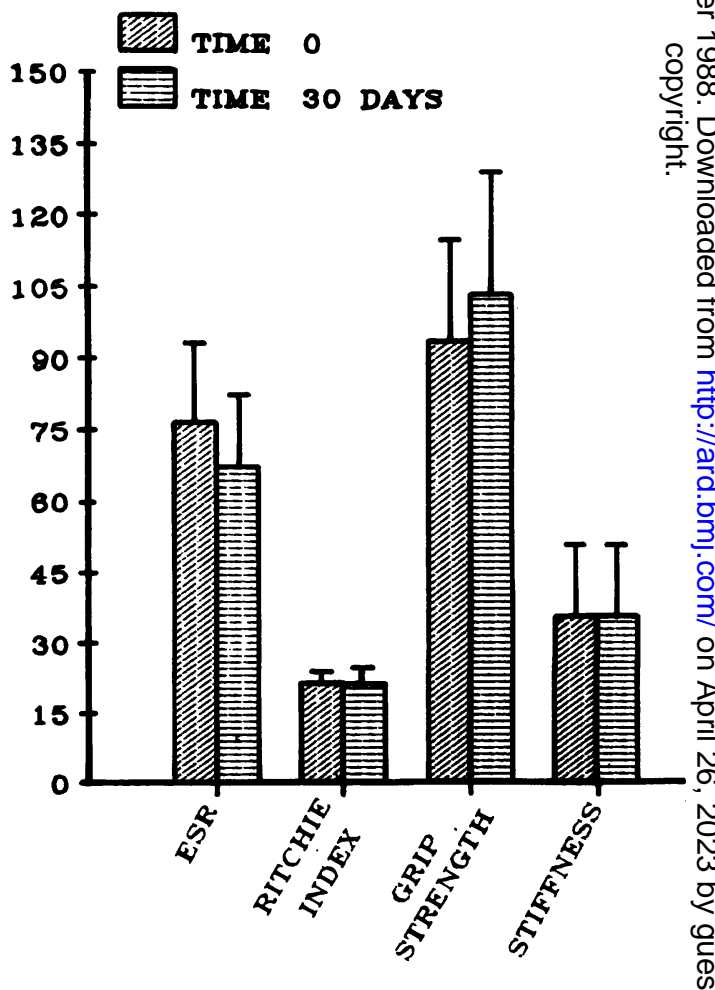

Fig. 1 Erythrocyte sedimentation rate $(E S R)(\mathrm{mm} / \mathrm{lst} \mathrm{h})$, Ritchie's index, grip strength ( $\mathrm{mmHg}$ ), and morning stiffness (minutes) at the start and after 30 minutes in group $\stackrel{\vec{D}}{\vec{d}}$ A patients. 

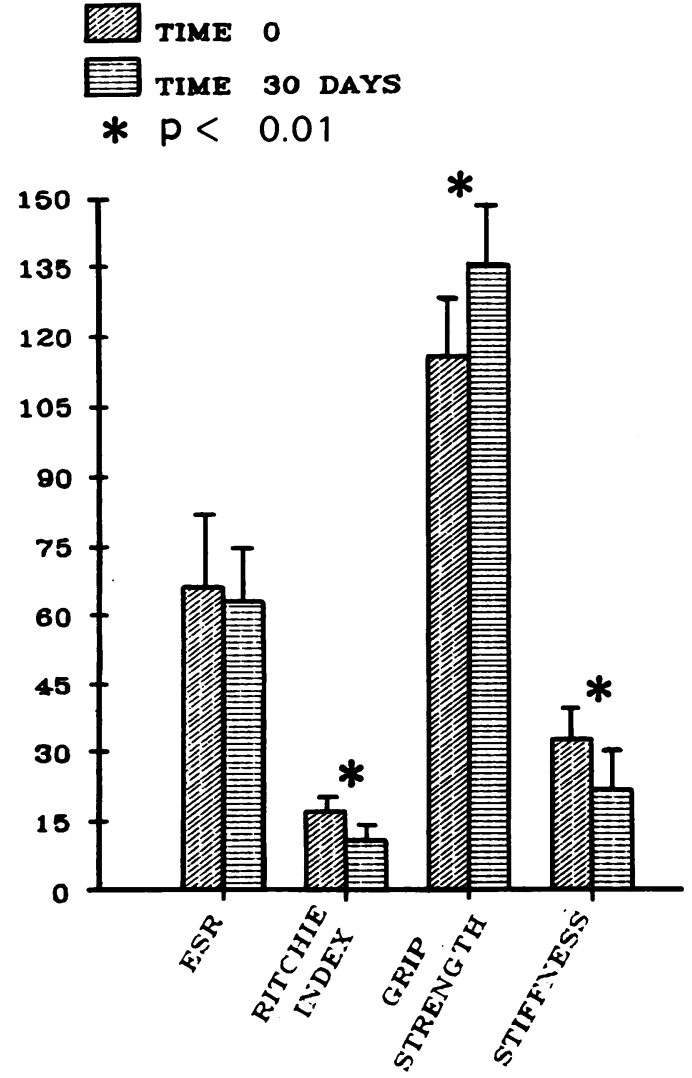

Fig. 2 ESR ( $\mathrm{mm} / 1 \mathrm{st} \mathrm{h})$, Ritchie's index, grip strength $(\mathrm{mmHg})$, and morning stiffness (minutes) at the start and after 30 minutes in group $B$ patients.

$v 36(10 \cdot 17) \mathrm{min} ; \mathrm{p}<0.01)$, and in grip strength (136 $(12.88)$ v $104(21.58) \mathrm{mmHg} ; \mathrm{p}<0.01)$.

Fig. 3 shows the chemiluminescence specific activities both for the patients receiving Max Epa and those on a diet high in saturated fatty acids. Patients treated with the diet high in saturated fatty acids showed a non-significant alteration in neutrophil chemiluminescence after 30 days $(2.66(0.38)$ $v 3.25(0.58) \mathrm{cpm} / \mathrm{PMN} ; \mathrm{p}=\mathrm{NS})$. In contrast, the Max Epa diet produced a notable reduction of neutrophil chemiluminescence after 30 days (2.04 $(0.3)$ v $3.67(0.87) \mathrm{cpm} / \mathrm{PMN}$; $\mathrm{p}<0.05)$.

No statistically significant changes in sedimentation rate, haemoglobin concentration, triglyceride, or cholesterol dietary levels were observed between the two dietary regimens.

\section{Discussion}

Alterations in essential fatty acids have been shown

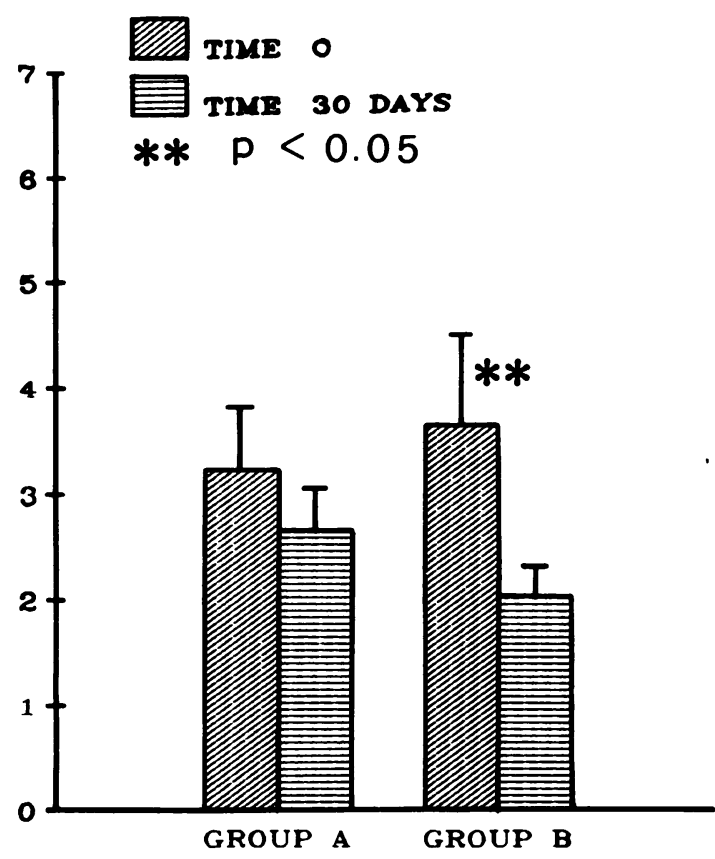

Fig. 3 Neutrophil chemiluminescence (obtained by dividing the maximum chemiluminescence activity by the number of neutrophils) at the start and after 30 days in group $A$ and group $B$ patients.

to affect immune function and the inflammatory response in animal models by various mechanisms. ${ }^{14} 15$ The cyclo-oxygenase and lipoxygenase products of n-3 fatty acids are less proinflammatory than those derived from arachidonic and other $n-6$ fatty acids, ${ }^{10} .16$ suggesting a possible anti-inflammatory role for fish oil. Eicosapentaenoic acid is a precursor for the 5 series of leucotrienes (LTB), and when the diet of rats is supplemented with this fatty acid there is a significant increase in the production of $\mathrm{LTB}_{5}$, which is 30 times less potent than $\mathrm{LTB}_{4}$ in causing neutrophil activation. ${ }^{16}$

The effect of a 30 day Max Epa diet on neutrophil chemiluminescence (Fig. 3) is clearly evident. Because the chemiluminescence in our system is dependent on a hydrophobic chemilumigenic probe (luminol), the decrease after a Max Epa diet is probably due to a change in neutrophil membrane lipid composition.

These results are in agreement with those of Lee et $a l,{ }^{10}$ who found a reduction of $\mathrm{LTB}_{4}$ and of chemotactic activity with an increase of membrane polarisation value in leucocytes of normal subjects receiving Max Epa.

The favourable effects observed with the administration to patients with RA of a diet high in 
polyunsaturated fatty acids derived from fish oil are in agreement with the results of Kremer et al, ${ }^{17}{ }^{18}$ who demonstrated a subjective alleviation of active RA and a reduction in neutrophil $\mathrm{LTB}_{4}$ production in these patients.

It is noteworthy that patients treated with a Max Epa diet were in a more severe class of RA (fewer in ARA class I-Table 1). This could mean that the improvement in this group of patients was even more significant.

Diets high in polyunsaturated fatty acids containing $n-3$ acids are generally well tolerated and produce a statistically significant reduction of disease activity. ${ }^{1617}$

The improvement noted in each clinical variable studied and in neutrophil chemiluminescence may be attributed to the same factors: cyclo-oxygenase and lipoxygenase products of $n-3$ fatty acids, which are less proinflammatory than those derived from arachidonic and other $n-6$ acids.

Further studies will, however, be necessary for a better understanding of the precise mechanism of action of fish oil supplementation in patients with RA.

\section{References}

1 Homsy J, Morrow W J W, Levy J A. Nutrition and autoimmunity: a review. Clin Exp Immunol 1986; 65: 473-88.

2 Gordon D J, Salz K M, Roggenkamp K J, Franklin F A Jr. Dietary determinants of plasma cholesterol change in the recruitment phase of the Lipid Research Clinics Coronary Primary Prevention Trial. Arteriosclerosis 1982; 2: 537-48.

3 Keys A, Anderson J T, Grande F. Serum cholesterol responses to changes in the diet. Metabolism 1965; 14: 747-87.

4 Nestel P J. Polyunsaturated fatty acids (n-3, n-6). Am J Clin Nutr 1987; 45: 1161-7.
5 Weisweiler P, Janetschek P, Schwandt P. Influence of polyunsaturated fats and fat restriction on serum lipoproteins in $\bar{\omega}$. humans. Metabolism 1985; 34: 83-6.

6 Needleman P, Raz A, Minkes M S, Ferrendelli J A, Sprecher $\overrightarrow{\overline{\vec{F}}}$ H. Triene prostaglandins:prostacyclin and thromboxane biosynthesis and unique biological properties. Proc Natl Acad SciC USA 1979; 76: 944-8.

7 Mansson I, Norberg R, Olhagen B, Bjorklund N E. Arthritis in pigs induced by dietary factors. Clin Exp Immunol 1971; 9: 677-93.

8 Darlington L G, Ramsey N W, Mansfield J R. Placebo-n controlled blind study of dietary manipulation therapy in rheumatoid arthritis. Lancet 1986; i: 236-8.

9 Davidson E M, Rae S A, Smith M J H. Leukotriene B4 a mediator of inflammation present in synovial fluid in rheuma- $\omega$ toid arthritis. Ann Rheum Dis 1983; 42: 677-9.

10 Lee T H, Hoover R L, Williams J D, et al. Effect of dietary enrichment with eicosapentaenoic and docosahexaenoic acids on in vitro neutrophil and monocyte leukotriene generation and neutrophil function. New Engl J Med 1985; 312: 1217-24.

11 Allen R C. Phagocytic leukocyte oxygenation activities and chemiluminescence: a kinetic approach to analysis. In: De Lucaco M A, MacElroy W D, eds. Methods in enzymology. Vol 33. Bioluminescence and chemiluminescence. New York: Academic음 Press, 1986: 449-93.

12 De Sole P, Lippa S, Littarru G P. Whole blood chemiluminescence: a new technical approach to assess oxygen-dependento microbicidal activity of granulocytes. Journal of Clinica $\bar{D}$ Laboratory Automation 1983; 3: 391-400.

13 Ropes M W, Bennett G A, Cobb S, Jacox R, Jessar R A. Revision of diagnostic criteria for rheumatoid arthritis. Bglke Rheum Dis 1958; 9: 175-6.

14 Good R A, West A, Fernandes G. Nutritional modulationimmune responses. Fed Proc 1980; 39: 3048-104.

15 Terano T, Salmon J A, Moncada S. Effect of orally administered eicosapentaenoic acid (EPA) on the formation of leukotriene B4 and leukotriene B5 by rat leukocytes. Biochem Pharmacol 1984; 33: 3071-6.

16 Kremer J M, Bigaouette J, Michalek A V, et al. Effects of manipulation of dietary fatty acids on clinical manifestations of rheumatoid arthritis. Lancet 1985; i: 184-7.

17 Kremer J M, Jubiz W, Michalek A, et al. Fish oil fatty acid supplementation in active rheumatoid arthritis. Ann Intern Med 1987; 106: 497-503. 